Article

\title{
Automated Mobile System for Accurate Outdoor Tree Crop Enumeration Using an Uncalibrated Camera
}

\author{
Thuy Tuong Nguyen ${ }^{1, *}$, David C. Slaughter ${ }^{2, *}$, Bradley D. Hanson ${ }^{3}$, Andrew Barber ${ }^{2}$, \\ Amy Freitas ${ }^{2}$, Daniel Robles ${ }^{2}$ and Erin Whelan ${ }^{2}$ \\ 1 Department of Computer Science, University of California, Davis, CA 95616, USA \\ 2 Department of Biological and Agricultural Engineering, University of California, Davis, \\ CA 95616, USA; E-Mails: abarber@ucdavis.edu (A.B.); asfreitas@ucdavis.edu (A.F.); \\ drrobles@ucdavis.edu (D.R.); emwhelan@ucdavis.edu (E.W.) \\ 3 Department of Plant Sciences, University of California, Davis, CA 95616, USA; \\ E-Mail: bhanson@ucdavis.edu \\ * Authors to whom correspondence should be addressed; E-Mails: thnguyen@ucdavis.edu (T.T.N.); \\ dcslaughter@ucdavis.edu (D.C.S.); Tel.: +1-530-752-5553 (T.T.N.); +1-530-752-5553 (D.C.S.); \\ Fax: +1-530-752-2640 (D.C.S.).
}

Academic Editor: Gonzalo Pajares Martinsanz

Received: 31 May 2015 / Accepted: 20 July 2015 / Published: 28 July 2015

\begin{abstract}
This paper demonstrates an automated computer vision system for outdoor tree crop enumeration in a seedling nursery. The complete system incorporates both hardware components (including an embedded microcontroller, an odometry encoder, and an uncalibrated digital color camera) and software algorithms (including microcontroller algorithms and the proposed algorithm for tree crop enumeration) required to obtain robust performance in a natural outdoor environment. The enumeration system uses a three-step image analysis process based upon: (1) an orthographic plant projection method integrating a perspective transform with automatic parameter estimation; (2) a plant counting method based on projection histograms; and (3) a double-counting avoidance method based on a homography transform. Experimental results demonstrate the ability to count large numbers of plants automatically with no human effort. Results show that, for tree seedlings having a height up to $40 \mathrm{~cm}$ and a within-row tree spacing of approximately $10 \mathrm{~cm}$, the algorithms successfully estimated the number of plants with an average accuracy of $95.2 \%$ for trees within a single image and $98 \%$ for counting of the whole plant population in a large sequence of images.
\end{abstract}


Keywords: tree crop enumeration; plant counting; uncalibrated camera; perspective transform; projection histogram; homography transform

\section{Introduction}

To help cope with the rapid increase in the human population and future demands on worldwide food security, automation in agriculture is necessary. For example, there is a need to develop automatic systems for plant enumeration in fruit and nut tree seedling crops to save human resources and improve yield estimation. Most sensors used in agriculture have limited resolution, and cannot acquire the full scope of available plant and soil information. Advanced sensors, like cameras, that can characterize spatial and color information of natural objects play a crucial role in the future development of agricultural automation [1-3].

In the fruit and nut tree nursery industry, accurate counts of tree seedlings are very important in their production management and commerce [4,5]. Disease resistant tree rootstocks are planted from seeds in an outdoor nursery and they are later grafted to have fruit, which is a different cultivar from the disease resistant cultivar of the root system (i.e., to combine the best features of two cultivars). Variability in germination rate and consumption by birds create uncertainty in the number of marketable seedlings available for sale. Traditionally, human workers must manually count the seedlings each spring after they have grown large enough to be safe from the birds and the final crop stand is stable. This method is slow, tedious, and costly for workers to perform. Additionally, while this method can be accurate when carefully conducted, in practice, human error and bias are still present and can lower the accuracy of the final count, particularly when workers get fatigued or distracted.

Recently, methods for plant population and spacing measurement using machine vision have been introduced for different kinds of plants. A daylight sensing system is presented in [4] to measure early growth stage corn populations. The algorithms used in the system include steps for image sequencing to merge information between consecutive video frames, vegetation segmentation using a truncated ellipsoidal decision surface and a Bayesian classifier, and plant counting based on the total number of plant pixels and their median positions. The image sequencing step in this study does not consider the case of a camera perspective change, however. In [5], algorithms for automatically measuring corn plant spacing at early growth stages are introduced. Plant morphological features, plant color, and the crop row centerline are among multiple sources of information utilized by the algorithms for corn plant detection and localization. This work points out that the estimated interplant spacing errors are due to crop damage and sampling platform vibration, which caused mosaicking errors. A machine vision-based corn plant spacing and population measurement system is presented in [6]. Algorithm steps in this paper include image sequencing using SIFT (Scale Invariant Feature Transform) feature matching, vegetation segmentation based on color channels, corn plant center detection using a skeletonizing algorithm, and calculation of corn spacing and plant count. This algorithm yields satisfactory results with images captured from the top view. In [7], we had proposed a mobile platform that utilizes active optical sensing devices (LIDAR and light curtain transmission) to detect, localize, and classify trees. Promising results in our recent work helped system designers select the most reliable 
sensor for accurate detection of trees in a nursery and to automate labor-intensive tasks, such as weeding, without damaging crops.

In recent years, high-resolution remote-sensing techniques have been utilized in agricultural automation for counting mature trees. An automatic approach for counting olive trees is introduced in [8] with very high spatial remote sensing images. This approach contains two main steps: The olive trees are first isolated from other objects in the image based on a Gaussian process classifier with different morphological and textural features; candidate blobs of olive trees are then considered valid if their sizes are in a range specified by a prior knowledge of the real size of trees. In [9], a method for counting palm trees in Unmanned Aerial Vehicles (UAV) images is presented. To detect palm trees, SIFT keypoints are extracted from the images, and then analyzed by an Extreme Learning Machine (ELM) classifier. The ELM classifier uses prior knowledge trained on palm and non-palm keypoints. The output of this step is a list of palm keypoints that are then merged using an active contour method to produce the shape of each tree. Local binary patterns are used to distinguish palm trees from other objects based on their shapes. A general image processing method for counting any tree in high-resolution satellite images is described in [10]. Steps used in this method include HSI (hue, saturation, intensity) color space transform of the original image, image smoothing, thresholding of the extracted hue image, histogram equalization for HSI channels, candidate region detection, delineation, and tree counting.

This paper presents an uncalibrated camera system for fast and accurate plant counting in an outdoor field and incorporates a single high quality camera, an embedded microcontroller to automate the image capturing process, and a computer vision algorithm. The algorithm includes the steps of orthographic plant projection based on a perspective transform, plant segmentation using excessive green, plant detection by utilizing projection histograms, and plant counting that compensates for overlapping areas between consecutive images (to avoid double-counting). Both the camera and microcontroller were mounted on an ATV (all-terrain vehicle) and the images were analyzed offline.

Compared to previously described systems, the advantages of the proposed system are: It is easy to setup without requiring any camera calibration, it is robust to shadows in the background (e.g., soil, plant residue, and plants in other rows) and, among the target plants, it powerfully copes with noise and foliage occlusion, and is suited for use on a mobile vehicle in the field where row paths are rough and camera vibration common. The ATV platform was used in this paper to simulate the normal orchard tractors that the system was designed to be mount on. The objective was to have a system that can be mounted on a tractor and the counting done as part of the existing field operations. The most compatible existing operation is the fertilization step that is done at the same time as the counting. Figure 1 shows two small tractors performing the fertilization step at the same time as plant counting. This step is compatible because the tractors travel each row and are generating minimal dust. It is also the main argument against a single purpose UAV because by adding a machine vision module onto the front of the tractor shown in Figure 1, we can also count plants in an existing trip of a ground vehicle that is traveling each row.

This paper is organized as follows: In Section 2, we describe the system design and the proposed algorithm. The algorithm is detailed in Sections 3 and 4 for plant counting for a single image and on an image sequence, respectively. Section 5 presents the experimental results of the system. Finally, this paper draws to a conclusion and discusses future work in Sections 6 and 7, respectively. 


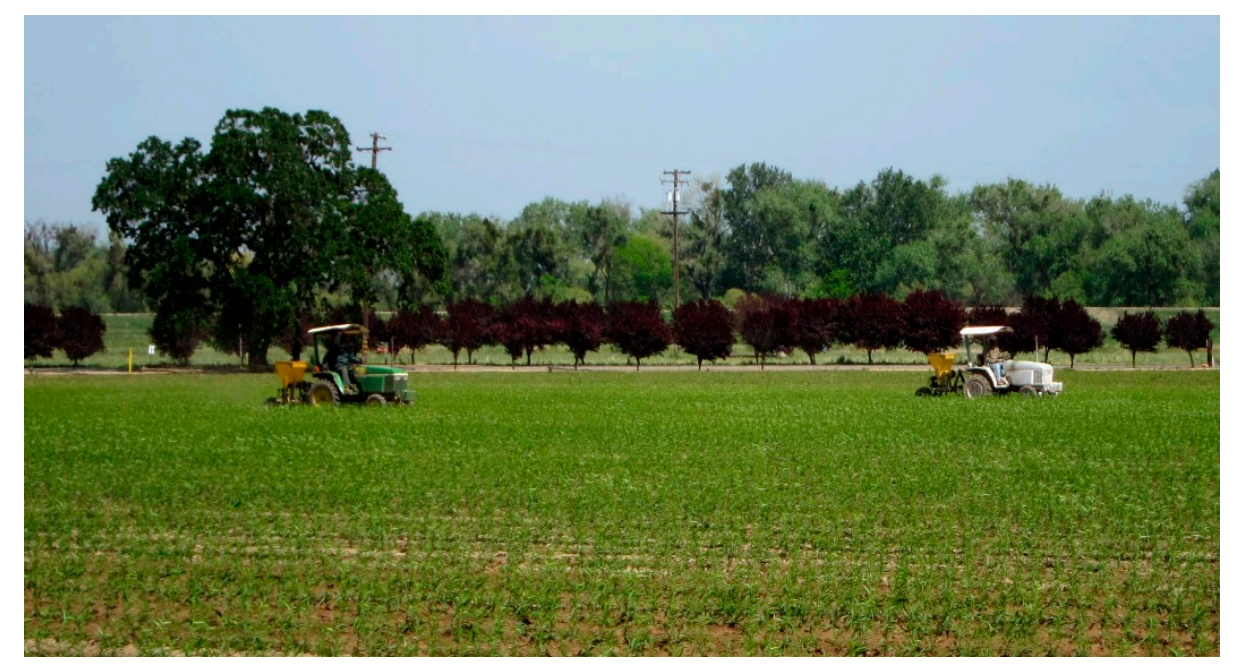

Figure 1. Tractors are performing the fertilization step at the same time as the counting, at Sierra Gold Nurseries, CA, USA.

\section{System Design and Algorithms}

In the experimental system an ATV was used to simulate a tractor, as the base platform. The mobile vehicle had an attached arm to hold a camera, and a rotary shaft encoder was mounted on the wheel axle for odometry sensing. Image data are based on 24-bit digital color images taken by an electrically controlled, high-resolution, digital single-lens reflex camera (model EOS Rebel T3, Canon Inc., Tokyo, Japan). The camera was equipped with a zoom lens (model EF-S 18-55 mm 1:3.5-5.6 IS II, Canon Inc., Tokyo, Japan) aimed at the target plants and held fixed on the arm mounted to the mobile vehicle at an orientation of approximately $60^{\circ}$ relative to the ground plane. An embedded microcontroller (model Raspberry Pi version 1 model B+, Raspberry Pi Foundation, UK) was used to activate the camera via a solid-state relay. The odometry signal was an input into the microcontroller to control the distance travelled between image acquisition events. A control algorithm was created to allow the microcontroller to trigger image acquisition by the camera at set spatial intervals. Because a difference in image resolution might dramatically affect the processing speed of the whole system from image transfer, plant segmentation, feature detection, and calculation of homography transformation matrix, the resolution $640 \times 480$ was selected as the best trade-off between speed and quality, so that accurate results can be obtained in an acceptable length of processing time. All camera parameters, including aperture, focal length, shutter speed, white balance, and ISO were manually set to have the best quality images in an outdoor scene. Figure 2 shows all details of the devices on the ATV, including the arm to mount the camera, the microcontroller with a relay and the wheel encoder.

The algorithm contains steps of perspective transform (with automatic determination of parameters) for orthographic plant projection, excessive green color segmentation, Gaussian smoothing, projection histogram, and local maxima detection for plant counting for a single image. To overcome potential problems with double-counting of plants in an image sequence, SURF (Speeded Up Robust Features) keypoint detection, SURF descriptor extraction [11], FLANN (Fast Library for Approximate Nearest Neighbors) descriptor matching [12], filtering of descriptor matches, and calculation of homography transform were utilized with GPU implementations. Figure 3 shows a block diagram of the proposed algorithm. 


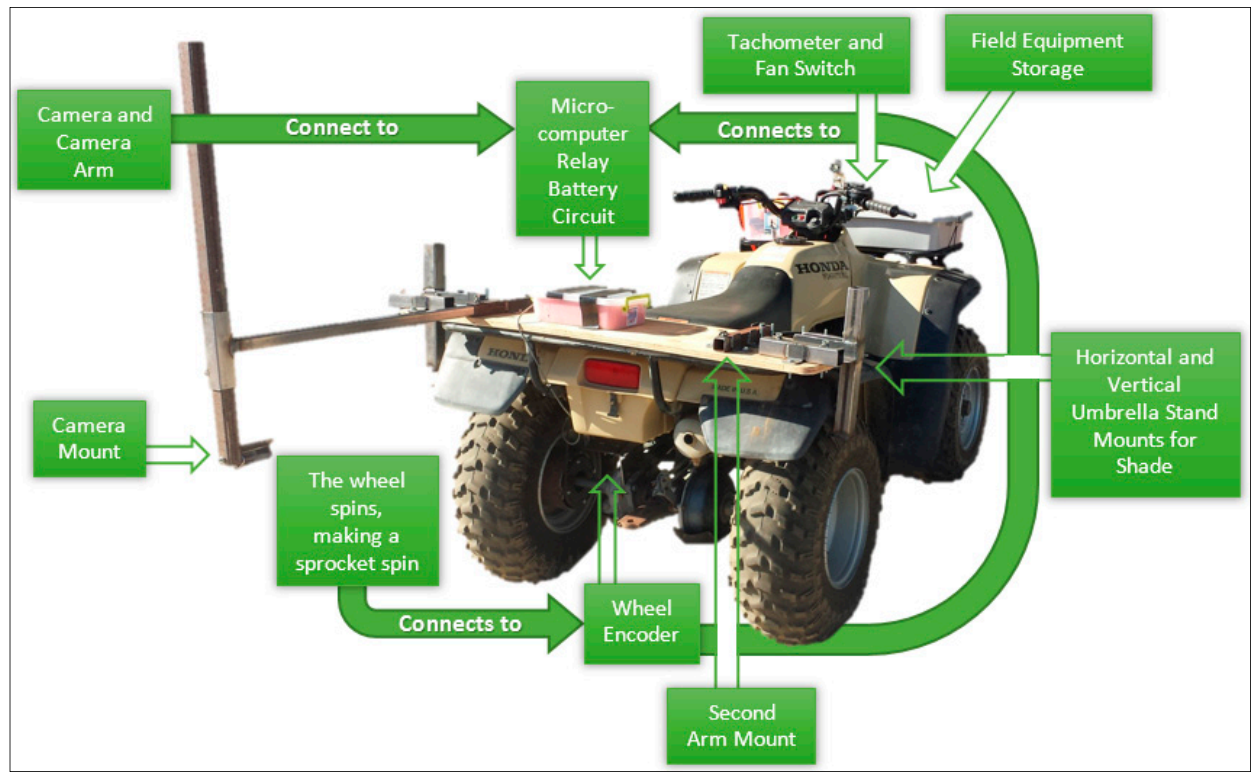

(a)

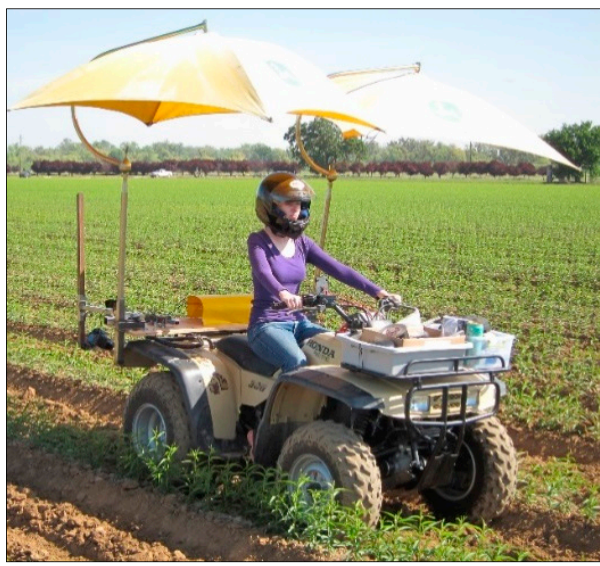

(b)

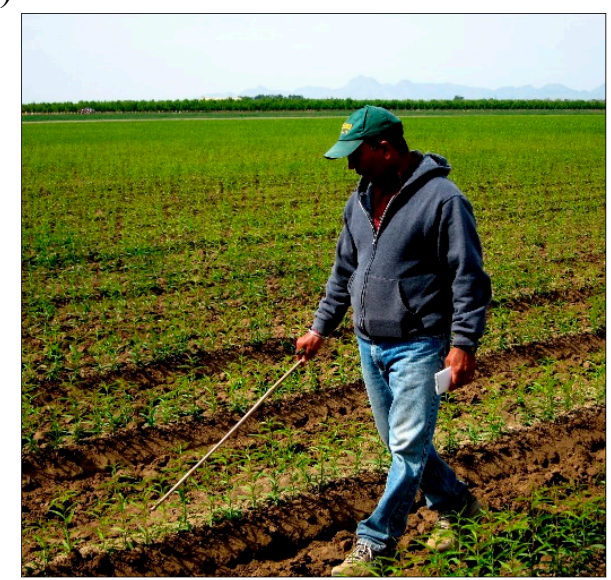

(c)

Figure 2. (a) The ATV with an arm to mount the camera, the wheel encoder, and the microcontroller to activate the camera via a relay; (b) the vehicle in operation capturing pictures of plants; and (c) manual counting of plants by a staff person.

Plant counting on a single image

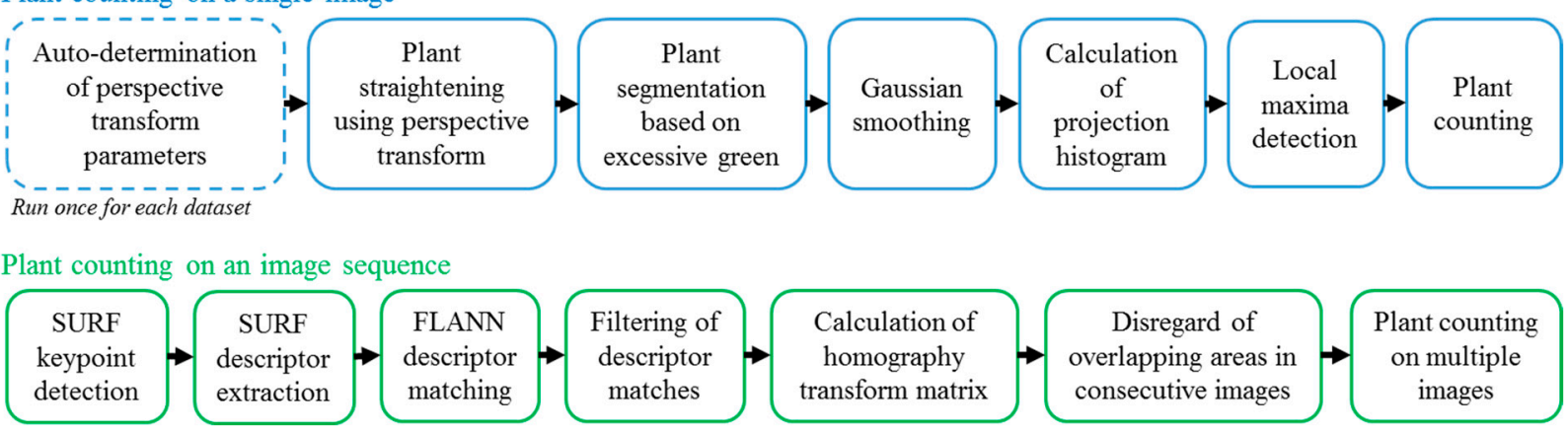

Figure 3. Flowchart representation of the algorithm. 


\section{Plant Counting in a Single Image}

\subsection{Plant Straightening Using a Perspective Transform}

Since the original image is collected using a perspective view, a correction method to straighten the plants (i.e., orthographic projection) for later processing in the steps of projection histogram calculation and local maxima detection is required. Existing studies $[13,14]$ have investigated the affine rectification of the ground plane to make parallel world lines appear parallel in the rectified image. Only one vanishing point is needed in these methods when the camera is at a fixed angle tilting downward toward the ground. This affine rectification method has proven robust to create a virtual orthographic view of the scene. The method can be successfully applied to our system if a vanishing point could be found; however, parallel world landmarks do not exist in the field and thus requires another way of transforming the image. In this section, a perspective transform technique is presented using four predefined points in the source image space and four in the destination image space. The $3 \times 3$ perspective transform matrix $\mathbf{P}$ is solved so that:

$$
\left[\begin{array}{c}
x_{i}^{\prime} \\
y^{\prime}{ }_{i} \\
1
\end{array}\right]=\mathbf{P}\left[\begin{array}{c}
x_{i} \\
y_{i} \\
1
\end{array}\right]=\left[\begin{array}{lll}
p_{11} & p_{12} & p_{13} \\
p_{21} & p_{22} & p_{23} \\
p_{31} & p_{32} & p_{33}
\end{array}\right]\left[\begin{array}{c}
x_{i} \\
y_{i} \\
1
\end{array}\right]
$$

where $i=\{1,2,3,4\} ;\left(x_{i}, y_{i}\right)$ and $\left(x^{\prime}{ }_{i}, y^{\prime}{ }_{i}\right)$ are the $i$-th points in the source and destination images, respectively. The four points in the source image are defined as

$$
\begin{gathered}
\left(x_{1}, y_{1}\right)=(0,0) \\
\left(x_{2}, y_{2}\right)=(W-1,0) \\
\left(x_{3}, y_{3}\right)=(W-1, H-1) \\
\text { and }\left(x_{4}, y_{4}\right)=(0, H-1)
\end{gathered}
$$

where $W$ and $H$ are the source image width and height, respectively. The four points in the destination image are calculated based on how the perspective changes with respect to $x$ and $y$ directions:

$$
\begin{gathered}
\left(x_{1}^{\prime}, y^{\prime}\right)=\left(\delta_{x}, \delta_{y}\right) \\
\left(x_{2}^{\prime}, y_{2}^{\prime}\right)=\left(\mathrm{W}-1-\delta_{x}, \delta_{y}\right) \\
\left(x_{3}^{\prime}, y_{3}^{\prime}\right)=\left(W-1+\delta_{x}, H-1+\delta_{y}\right) \\
\text { and }\left(x_{4}^{\prime}, y_{4}^{\prime}\right)=\left(-\delta_{x}, H-1+\delta_{y}\right)
\end{gathered}
$$

where $\delta_{x}$ and $\delta_{y}$ express how the perspective is needed to change (see Figure 4). These parameters can be determined automatically using our proposed algorithm that contains all steps from plant straightening to local maxima detection. The algorithm of auto-determination of perspective transform parameters is used only once on the first image of each dataset. The determined $\delta_{x}$ and $\delta_{y}$ are then applied to the remaining images in the set. This algorithm will be described in detail later in this section. Based on the obtained perspective transform matrix $\mathbf{P}$, the destination image is transformed by

$$
I_{p}(x, y)=I\left(\frac{p_{11} x+p_{11} y+p_{13}}{p_{31} x+p_{32} y+p_{33}}, \frac{p_{21} x+p_{22} y+p_{23}}{p_{31} x+p_{32} y+p_{33}}\right)
$$


where $I_{p}(x, y)$ and $I(x, y)$ are the destination and source images, respectively. Figure 4 shows a sample image of ten small peach trees before and after performing a perspective transform. In this example, the average inclination angle of plants, with respect to $x$-axis, is significantly corrected from $74.81^{\circ}$ to $82.77^{\circ}$.

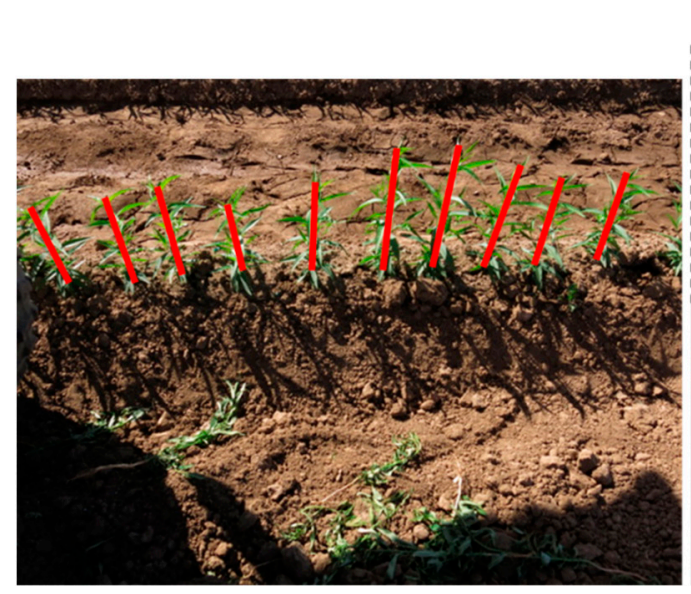

(a)

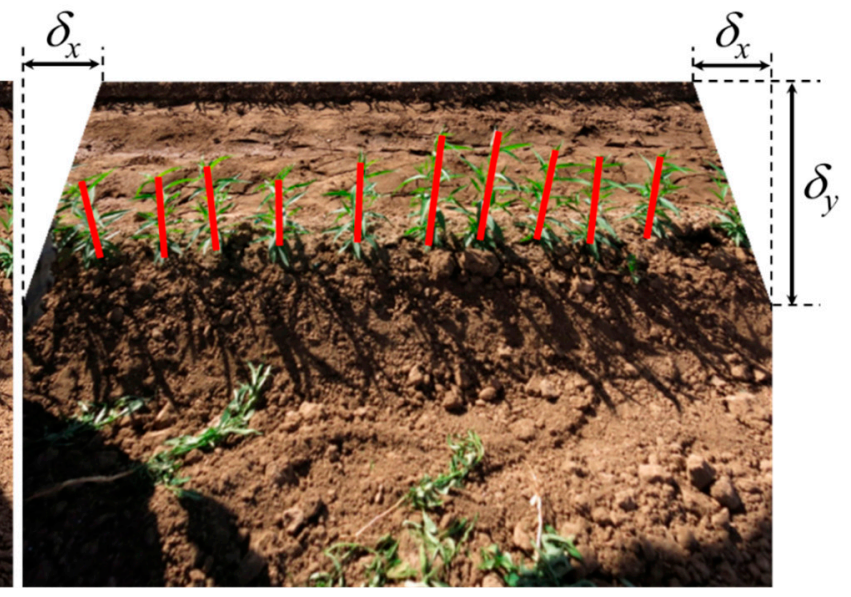

(b)

Figure 4. An image before (a) and after (b) performing a perspective transform for plant straightening. The (red) solid lines show the plant inclination. The average inclination angle (with respect to $x$-axis) of the ten plants in the original image (a) is $74.81^{\circ}$; and it is $82.77^{\circ}$ in the corrected one (b).

\subsection{Plant Segmentation Based on Excessive Green}

Different algorithms for soil, ground, and plant segmentation have been introduced in [15-17]. This step herein utilizes a fast and efficient method to segment green objects from the soil or background. The plants and the less-green background in the perspective transformed image were segmented by comparing the excessive green value [17] of each pixel to a certain threshold:

$$
G(x, y)=\left\{\begin{aligned}
I_{p}(x, y), & \text { if }\left(2 I_{p}^{g}(x, y)-I_{p}^{r}(x, y)-I_{p}^{b}(x, y)\right)>T_{g} \\
0, & \text { otherwise }
\end{aligned}\right.
$$

where $G(x, y)$ is the green-segmented image; $I_{p}^{g}, I_{p}^{r}$, and $I_{p}^{b}$ are the green, red, and blue image channels of $I_{p}(x, y)$, respectively; and $T_{g}$ is a predefined threshold, which was fixed to 40 in this paper. This green color segmentation technique is very fast and effective in our system in segmenting plants from the backgrounds of soil, shadow, or non-green objects. Gaussian smoothing was applied to the thresholded excessive green image to eliminate background noise and reduce the effects of foliage occlusion. In this paper, based on the maximum plant height (approximately $40 \mathrm{~cm}$ ) and the degree of foliage occlusion from plant to plant, the Gaussian kernel standard deviation size was fixed to 7 . Figure 5 presents the green-segmented image of the transformed image in Figure 4 and the corresponding smoothed image. 


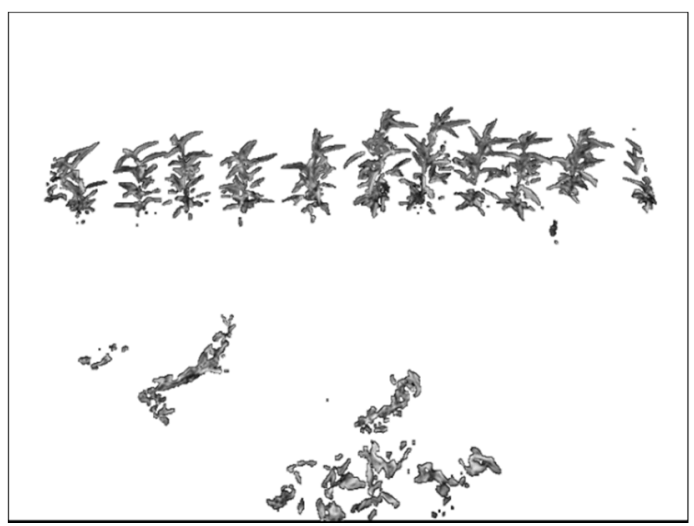

(a)

\section{$\begin{array}{llllllllll}1 & 2 & 3 & 4 & 5 & 6 & 7 & 8 & 9 & 10\end{array}$}

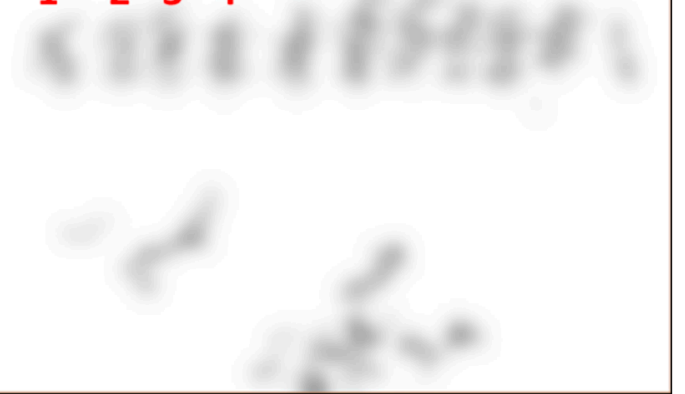

(b)

Figure 5. A green-segmented image (a) and its Gaussian smoothed image (b) where plants are isolated.

\subsection{Projection Histogram and Local Maxima Detection}

A vertical projection histogram [14] of the smoothed image is calculated in this section. It is constructed by projecting plant pixels along vertical lines (columns) in a region of interest (ROI). The number of bins in the histogram is equal to the number of columns in the image. In our system, because the camera is fixed on the mobile vehicle, we manually select the ROI to fit all plants in the field row. Let $P^{V}\left(G_{g}\right)$ be the vertical histogram of the smoothed image $G_{g}$, giving

$$
P^{V}\left(G_{g}\right)=\left\{h_{j}\left(G_{g}\right): j=0,1, \ldots, W-1\right\}
$$

Local maxima are detected by finding the histogram bins, where their gray values are greater than their neighbors as

$$
L(i)=\left\{h_{i}\left(G_{g}\right): h_{i-l}\left(G_{g}\right)<h_{i}\left(G_{g}\right) \text { and } h_{i+l}\left(G_{g}\right)<h_{i}\left(G_{g}\right), i=1,2, \ldots, W-2\right\}
$$

where $L(i)$ is the gray value of the local maximum at bin $i$. The number of plants is then determined based on the number of local maxima. It is noted that the detection of the plants at the left and right borders explicitly followed Equation (7) and the definition of ROI, although objects outside the ROI occasionally appear to human eyes, as at the right bottom edges of Figure 5 . The (red) numbers in Figure 5 represent the number of detected trees.

\subsection{Auto-Determination of Perspective Transform Parameters}

Notice that this step combines all steps from Sections 3.1-3.3 and it is done once on the first image of each dataset to have the optimal perspective transform parameters $\delta_{x}$ and $\delta_{y}$ for plant straightening and vertical histogram projection. The parameters $\delta_{x}$ and $\delta_{y}$ are estimated based on a list of detected local maxima as follows:

$$
\Delta\left(\delta_{x}, \delta_{y}\right)=\arg \max _{\substack{0 \leq \delta_{x} \leq W / 4 \\ 0 \leq \delta_{y} \leq H / 2}} \sum_{k=0}^{n_{L}} L(k)
$$


where $n_{L}$ is the number of local maxima and it is predefined as 10 for the image in Figure 5. This step particularly requires proper parameter tuning for the Gaussian filter to smooth the projection histogram and to have less noise in the detection of local maxima.

\section{Plant Counting in an Image Sequence}

Once the number of plants in each image is determined, the accumulated count in all images in a sequence is determined using a special method to avoid double-counting of trees between images. To serve this purpose, the homography transformation between every two successive images is considered. SURF is one of the best algorithms for keypoint detection and descriptor extraction and it is being used successfully in the applications of object recognition [18], image stitching [19], 3D reconstruction [20], and background motion compensation [21,22]. In this paper, SURF was utilized for the calculation of the homography transformation matrix, because it is fast to compute and has good performance when based on integral images and an integer approximation of the determinant of Hessian blob detector. The extracted SURF descriptors are matched between two consecutive images using FLANN, which allows fast and accurate nearest neighbor searches in high dimensional spaces. Furthermore, it can select the optimal matching parameters automatically without any tuning from users. Good matches are thenceforth obtained based on the condition of common distance, i.e., the matches having distances less than a minimum distance threshold will be discarded. In our case, a vehicle is moving along a field row and taking pictures of plants, two consecutive images of the same planar ground surface can be related by homography. The good matches obtained are used as the input to find a homography transformation between each set of successive images, without the need for camera calibration. Once the homography transformation matrix is found, camera translation with respect to vehicle's horizontal movement can be extracted to estimate the overlap between successive images and avoid double-counting of plants. Defining $I_{t-1}(x, y)=\left[x_{t-1}, y_{t-1}, 1\right]^{T}$ and $I_{t}(\mathrm{x}, \mathrm{y})=\left[x_{t}, y_{t}, 1\right]^{T}$ the image points as time $t-1$ and $t$, a homography $\mathbf{H}$ is represented through

$$
\left[\begin{array}{c}
x_{t} \\
y_{t} \\
1
\end{array}\right]=\mathbf{H}\left[\begin{array}{c}
x_{t-1} \\
y_{t-1} \\
1
\end{array}\right]=\left[\begin{array}{lll}
h_{11} & h_{12} & h_{13} \\
h_{21} & h_{22} & h_{23} \\
h_{31} & h_{32} & h_{33}
\end{array}\right]\left[\begin{array}{c}
x_{t-1} \\
y_{t-1} \\
1
\end{array}\right]
$$

where $h_{13}$ is the coefficient of $x$-translation and that is the parameter we want to utilize for estimating the overlapping between successive images. It is noted that the scaling parameters $h_{11}$ and $h_{22}$ approach 1 because there are no zoom-in or zoom-out operators from the camera and the vehicle is travelling along the plant row. In our case, an affine homography is considered an appropriate model of image displacement and is a special type of a general homography where $h_{31}$ and $h_{32}$ are close to 0 and $h_{33}$ approaches 1. In this paper, the Hessian threshold for the keypoint detector was fixed to 400, i.e., the features having a Hessian value larger than this threshold are retained. A RANSAC-based method was used to estimate the homography matrix so as to

$$
\begin{array}{r}
\mathbf{H}=\arg \min _{\mathbf{H}} \sum_{i=0}^{N}\left[\left(x_{t}^{i}-\frac{h_{11} x_{t-1}^{i}+h_{12} y_{t-1}^{i}+h_{13}}{h_{31} x_{t-1}^{i}+h_{32} y_{t-1}^{i}+h_{33}}\right)^{2}\right. \\
\left.+\left(y_{t}^{i}-\frac{h_{21} x_{t-1}^{i}+h_{22} y_{t-1}^{i}+h_{23}}{h_{31} x_{t-1}^{i}+h_{32} y_{t-1}^{i}+h_{33}}\right)^{2}\right]
\end{array}
$$


where $N$ is the total number of keypoints and $\left(x_{t}^{i}, y_{t}^{i}\right)$ is the image point $i$ at time $t$. Local maxima positions of plants are then compared to the $x$-translation parameter $h_{13}$ to determine whether they are double-counted. Due to the effect of camera distortion (i.e., the plants at the image border are less straight than those closer to image center), this determination step requires a "buffer" for the plants at the image border to reduce the amount of counting errors. When $h_{13}>0$, the comparison $\left(i l-b>h_{13}\right)$ implies that the plant at the local maxima position $i_{l}$ (obtained from the Equation (7)) is considered double-counted if its difference from the buffer $b$ is greater than $h_{13}$. When $h_{13} \leq 0$, the comparison $\left(i_{l}+b<W+h_{13}\right)$ is to check if the plant at $i_{l}$ is double-counted, where $W$ is image width. The buffer $b$ can be estimated automatically based on half of the average of distances between two neighbor local maxima.

\section{Experimental Results}

A computer (CPU model Core i7 at $3.4 \mathrm{GHz}$, Intel Co., Santa Clara, CA, USA, with 12-GB DDR3 random-access-memory) was used for all processing steps, except that an 1152 core GPU (model GeForce GTX 760, NVidia Co., Santa Clara, CA, USA) graphics card was utilized for implementing the GPU-based SURF descriptor extraction and FLANN matching algorithms. Experiments were conducted on 941 images containing 9915 juvenile Prunus persica L. "Nemaguard" peach trees (approximately 10.54 plants per image). Accounting for image overlap, the 941 images contained 2178 distinct peach trees when double-counting was eliminated. The images of the juvenile peach trees were collected from seven rows, with a $10 \mathrm{~cm}$ in-row plant distance between two neighboring plants, in an outdoor tree nursery (Sierra Gold Nurseries, Yuba City, CA, USA). Table 1 shows information for the datasets used in the experimental results, where Sets 1,2, and 3 (namely, Group 1) were taken on the same day and Sets 4 to Set 7 (namely, Group 2) were acquired on another day. The maximum plant height was approximately $30 \mathrm{~cm}$, and $40 \mathrm{~cm}$ in Groups 1 and 2, respectively. The overlap between two consecutive images was increased from approximately $60 \%$ in Group 1 to approximately $90 \%$ in Group 2 . These plant images were selected as examples of plants covered by shadow (from human, other plants, or random objects), green and other objects in the background, small plants, and different plant sizes.

Figure 6 presents sample images showing examples of the green plant residue between the rows of plants, a second row of trees at the back (Figure 6a) and eight small plants (Figure 6b). In this study, the ground truth number of plants and overlap of images were measured manually. The number of small plants was defined based on a maximum plant height of $15 \mathrm{~cm}$. It is noted that there were no plants imaged in shadow from Sets 3 to 7, and no additional objects on the background in Set 3. In the final design of our system on a tractor, a metal tunnel will be used to eliminate the current issues of shadows and green objects (i.e., the adjacent row) in the background. The purpose of presenting results of these critical issues here was to experimentally show the robustness of the proposed algorithm. In the experiments, all parameters were fixed (i.e., not optimized image by image) in order to have consistent results between different images and datasets. 
Table 1. List of datasets and their detail information for experiments.

\begin{tabular}{|c|c|c|c|c|c|c|c|c|c|}
\hline & & Set 1 & Set 2 & Set 3 & Set 4 & Set 5 & Set 6 & Set 7 & Total \\
\hline \multirow{5}{*}{ \# images } & All & 121 & 127 & 93 & 150 & 150 & 150 & 150 & 941 \\
\hline & Containing shadows & 18 & 3 & 0 & 0 & 0 & 0 & 0 & 21 \\
\hline & $\begin{array}{c}\text { Containing green } \\
\text { material on the } \\
\text { background }\end{array}$ & 6 & 10 & 46 & 150 & 150 & 150 & 150 & 662 \\
\hline & $\begin{array}{c}\text { Containing other } \\
\text { objects on the } \\
\text { background }\end{array}$ & 3 & 5 & 0 & 13 & 13 & 6 & 9 & 49 \\
\hline & $\begin{array}{c}\text { Containing small } \\
\text { plants }\end{array}$ & 18 & 21 & 12 & 6 & 5 & 11 & 34 & 107 \\
\hline \multirow{4}{*}{ \# plants } & In individual images & 1469 & 1481 & 1154 & 1398 & 1402 & 1505 & 1506 & 9915 \\
\hline & In the image sequence & 609 & 505 & 525 & 160 & 136 & 94 & 149 & 2178 \\
\hline & $\begin{array}{c}\text { With shadows in } \\
\text { individual images }\end{array}$ & 46 & 7 & 0 & 0 & 0 & 0 & 0 & 53 \\
\hline & $\begin{array}{c}\text { Of small size in } \\
\text { individual images }\end{array}$ & 26 & 42 & 13 & 6 & 5 & 15 & 53 & 160 \\
\hline Avel & image overlap & $59.2 \%$ & $64.7 \%$ & $53.8 \%$ & $89.4 \%$ & $91.1 \%$ & $93.8 \%$ & $90.4 \%$ & \\
\hline
\end{tabular}

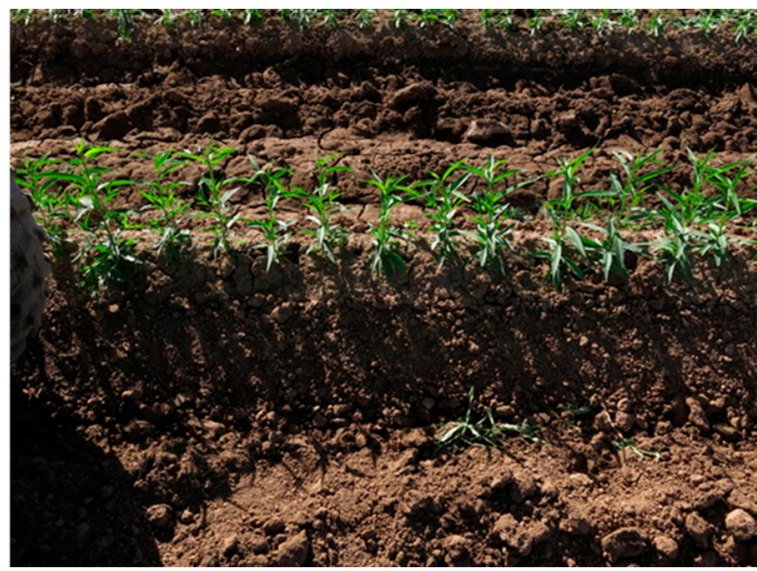

(a)

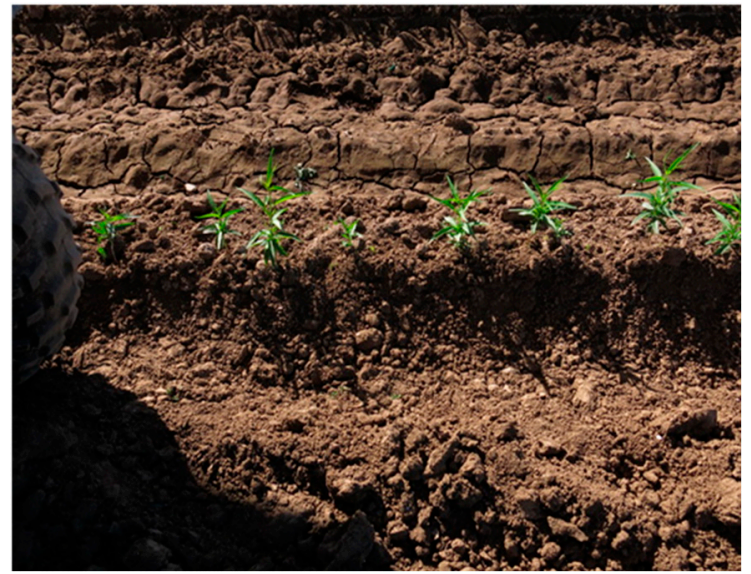

(b)

Figure 6. Sample images of green objects in the background (a) where there is plant residue on the soil and plants in the next row; and small plants (b).

Table 2 shows the average plant counting accuracies in both plant counting within a single image and for an image sequence, where double-counting was avoided. Within a single image, on average 0.51 counting mistakes out of 10.54 plants per image (i.e., an average accuracy of $95.2 \%$ ) were observed. Accuracies obtained when excluding the challenging cases $95.4 \%$ (shadows), 95.4\% (additional green objects), $95.3 \%$ (other objects), and $95.3 \%$ (small plants) were approximately equivalent to the overall performance, demonstrating the robustness of the method. Notice that the underlined numbers in Table 2 for the cases of "shadow" and "green objects" were taken from those without the exclusion. In an image sequence, when double-counting was minimized, an accuracy of $98 \%$ in all images was achieved. This accuracy is better than the single image performance because, on average, errors in a sequence of 
overlapped images compensate for one another. The plant counting error in every image is shown in Figure 7. The maximum estimation error was five plants and was mostly associated with high levels of foliage occlusion. By estimating the homography transformation between consecutive images, the proposed system also produced knowledge on the amount of image overlap. Comparing the actual overlap to that estimated by homography, we obtained an average error of only $2.54 \%$. The average processing time for all steps in the software algorithm was approximately $300 \mathrm{~ms}$ per image pair.

Table 2. Average counting accuracy and the estimated image overlap using a homography transform.

\begin{tabular}{|c|c|c|c|c|c|c|c|c|c|}
\hline & & Set 1 & Set 2 & Set 3 & Set 4 & Set 5 & Set 6 & Set 7 & Avg.* \\
\hline \multirow{7}{*}{$\begin{array}{l}\text { Within } \\
\text { single } \\
\text { images }\end{array}$} & $\begin{array}{l}\text { w.r.t. individual } \\
\text { images }\end{array}$ & $95.8 \%$ & $95.5 \%$ & $96.4 \%$ & $93.8 \%$ & $93.6 \%$ & $95.8 \%$ & $95.3 \%$ & $95.2 \%$ \\
\hline & $\begin{array}{l}\text { Avg. count errors } \\
\text { per image }\end{array}$ & 0.51 & 0.52 & 0.44 & 0.57 & 0.60 & 0.42 & 0.47 & 0.51 \\
\hline & $\begin{array}{l}\text { Std. Dev. of count } \\
\text { errors per image }\end{array}$ & 0.82 & 0.67 & 0.67 & 0.74 & 0.83 & 0.59 & 0.63 & 0.72 \\
\hline & $\begin{array}{c}\text { Excluding the case } \\
\text { of shadows }\end{array}$ & $96.2 \%$ & $95.8 \%$ & $\underline{96.4 \%}$ & $\underline{93.8 \%}$ & $\underline{93.6 \%}$ & $\underline{95.8 \%}$ & $\underline{95.3 \%}$ & $95.4 \%$ \\
\hline & $\begin{array}{l}\text { Excluding the case } \\
\text { of green objects on } \\
\text { the background }\end{array}$ & $95.7 \%$ & $95.5 \%$ & $96.7 \%$ & - & - & - & - & $95.4 \%$ \\
\hline & $\begin{array}{l}\text { Excluding the case } \\
\text { of other objects on } \\
\text { the background }\end{array}$ & $95.6 \%$ & $95.6 \%$ & $\underline{96.4 \%}$ & $93.9 \%$ & $93.7 \%$ & $96.1 \%$ & $95.8 \%$ & $95.3 \%$ \\
\hline & $\begin{array}{l}\text { Excluding the case } \\
\text { of small plants }\end{array}$ & $95.8 \%$ & $95.4 \%$ & $96.5 \%$ & $94.0 \%$ & $93.9 \%$ & $96.2 \%$ & $95.4 \%$ & $95.3 \%$ \\
\hline \multicolumn{2}{|c|}{ For an image sequence } & $99.2 \%$ & $98.2 \%$ & $99.2 \%$ & $96.3 \%$ & $95.6 \%$ & $99 \%$ & $98.6 \%$ & $98 \%$ \\
\hline \multicolumn{2}{|c|}{ Estimated image overlap } & $57.1 \%$ & $62.1 \%$ & $58.7 \%$ & $87.6 \%$ & $88.6 \%$ & $91.7 \%$ & $88.6 \%$ & \\
\hline
\end{tabular}

*: The final average values were calculated across all datasets.

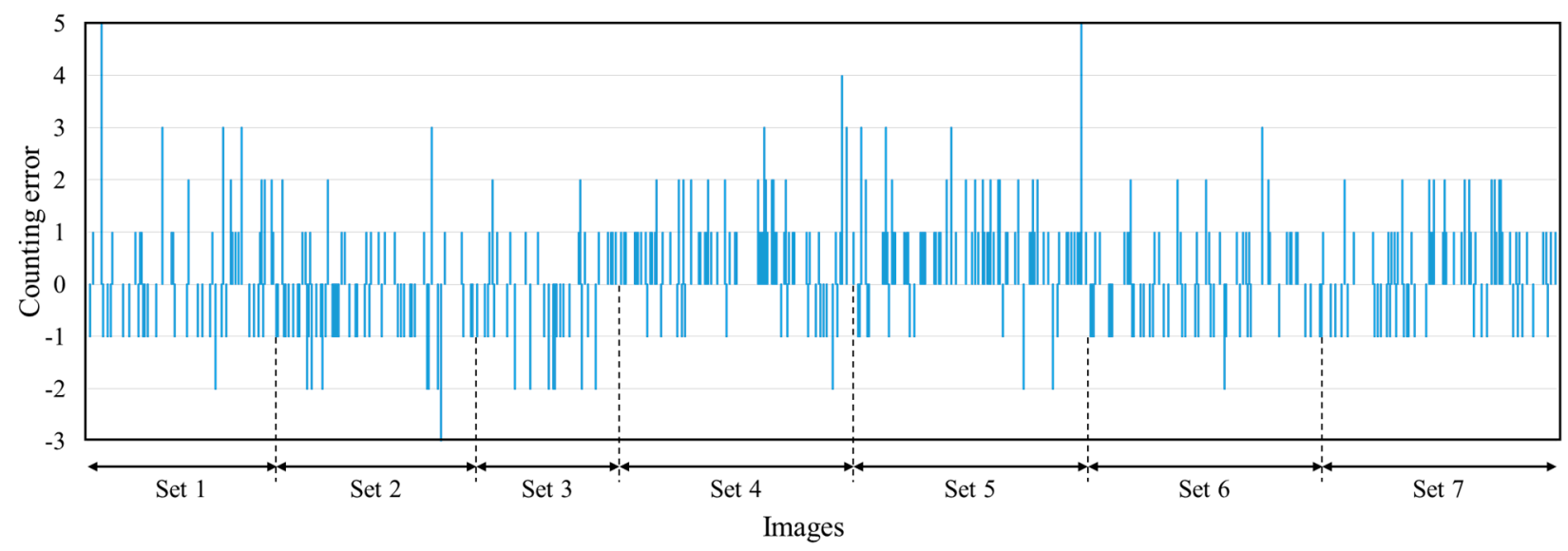

Figure 7. Tree counting errors in 941 images.

Comparisons in terms of counting accuracy and system characteristics between our method and the other two methods in [4,6] (where corn plants were used) are shown in Table 3 . The results are presented with respect to counting in individual images, and for an image sequence using our peach tree data. In 
the method [4], the block matching based image sequencing algorithm was not applicable to our data, which were captured from a camera held at an angle of $60^{\circ}$. Due to a large number of incorrect matches yielded by the block matching based algorithm, our image sequencing algorithm (without the perspective transform) was substituted for the block matching in [4] to allow comparison of subsequent steps. The accuracies yielded by [4] were significantly lower than ours. The iterative rules based on the number of pixels and positions were sensitive to plant size and plant center locations. Additionally, these rules required parameter tuning for refining plant and background regions. Similarly, in the method from [6], when the top view was used, it led to plant counting errors when the direct image sequencing was utilized without the perspective transform. Using skeletonization for plant center detection, the method in [6] yielded a high error rate when there was foliage occlusion. For individual images, our system yielded an average accuracy of $95.2 \%(0.51 \pm 0.72$ count errors per image $)$ compared to $86.9 \%(1.38 \pm 1.34)$ for the method of [4] and $71.4 \%(2.05 \pm 1.96)$ for the method in [6]. In the case of the image sequence, our total accuracy (98\%) was substantially better than those of the other two methods $(77.8 \%$ and $71.9 \%)$. It is worth mentioning that our method was able to work well with less than $60 \%$ image overlapping compared to $85 \%$ of [4].

Table 3. Accuracy and system characteristics comparison of the proposed method to $[4,6]$.

\begin{tabular}{|c|c|c|c|c|}
\hline & & The Method of [4] & The Method of [6] & The Proposed Method \\
\hline \multicolumn{5}{|c|}{ Accuracy comparison (using peach tree seedling data) } \\
\hline \multirow{3}{*}{$\begin{array}{l}\text { Within } \\
\text { individual } \\
\text { images }\end{array}$} & $\begin{array}{l}\text { w.r.t. individual } \\
\text { images }\end{array}$ & $86.9 \%$ & $71.4 \%$ & $95.2 \%$ \\
\hline & $\begin{array}{l}\text { Avg. count errors } \\
\text { per image }\end{array}$ & 1.38 & 2.05 & 0.51 \\
\hline & $\begin{array}{l}\text { Std. Dev. of count } \\
\text { errors per image }\end{array}$ & 1.34 & 1.96 & 0.72 \\
\hline \multicolumn{2}{|c|}{ In an image sequence } & $77.8 \%$ & $71.9 \%$ & $98 \%$ \\
\hline \multicolumn{5}{|c|}{ System characteristics } \\
\hline \multicolumn{2}{|c|}{ Plant size (growth stage) } & V3 to V4 growth stages * & $\begin{array}{c}\text { Early to V3 } \\
\text { growth stages } *\end{array}$ & $\begin{array}{l}\text { Early growth stage to } \\
\qquad 40 \mathrm{~cm} \text { height }\end{array}$ \\
\hline \multicolumn{2}{|c|}{ Camera view } & Top view & Top view & $\begin{array}{c}\text { Perspective view at an } \\
\text { angle of } 60^{\circ}\end{array}$ \\
\hline \multicolumn{2}{|c|}{ Image overlap } & $85 \%$ & $\mathrm{n} / \mathrm{a}$ & $54 \%$ to $91 \%$ \\
\hline \multirow{3}{*}{ Method } & Image sequencing & $\begin{array}{c}\text { Block matching } \\
\text { (substituted by our image } \\
\text { sequencing method } \\
\text { without perspective } \\
\text { transform) }\end{array}$ & $\begin{array}{l}\text { SIFT feature matching, } \\
\text { homography transform }\end{array}$ & $\begin{array}{c}\text { SURF descriptor } \\
\text { extraction, RANSAC } \\
\text { feature matching, } \\
\text { homography transform }\end{array}$ \\
\hline & Plant segmentation & $\begin{array}{c}\text { Bayesian classification on } \\
\text { color spaces }\end{array}$ & $\begin{array}{c}\text { Bayesian classification } \\
\text { on color spaces }\end{array}$ & Excessive green \\
\hline & Plant counting & $\begin{array}{l}\text { Iterative rules based on the } \\
\text { number of pixels and } \\
\text { positions }\end{array}$ & $\begin{array}{l}\text { Skeletonization for } \\
\text { plant center detection }\end{array}$ & $\begin{array}{l}\text { Perspective transform, } \\
\text { Gaussian smoothing, } \\
\text { projection histogram }\end{array}$ \\
\hline
\end{tabular}

*: The V3 growth stage in corn implies three leaves with visible leaf collars. 


\section{Conclusions}

A digital imaging system for tree crop enumeration was successfully developed and tested. The system uses an embedded microcontroller mounted on an ATV (to simulate a tractor that will be used in actual practice the field) to receive the odometry signal and trigger the image acquisition. In this paper, the ability to automatically count a large number of small peach trees, with no human effort compared to manual counting, was demonstrated. The estimated count allows confirmation of the germination rate and final plant stand prior to budding in tree seedlings grown in outdoor nurseries. For the juvenile trees to a $40 \mathrm{~cm}$ height and $10 \mathrm{~cm}$ within-row plant spacing, the method successfully counted plants with an average accuracy of $95.2 \%$ in individual images and $98 \%$ for counting the whole plant population in a long image sequence. The method also provides robust performance for the cases of plants in shadow, green and other colored objects on the image background, small plants, and foliage occlusion.

\section{Future Work}

Future work should include (1) automating the system to perform real-time analysis and plant counting in the field; (2) improving and optimizing the algorithms to support larger seedlings; (3) counting multiple rows (Figure 8) simultaneously for a redundant check on the count or allowing a count with fewer passes through the field; (4) supporting measurements of stem width and plant height; and (5) plant phenotyping for tree crops in seedling nurseries and for mapping the location of each plant for individual plant care.

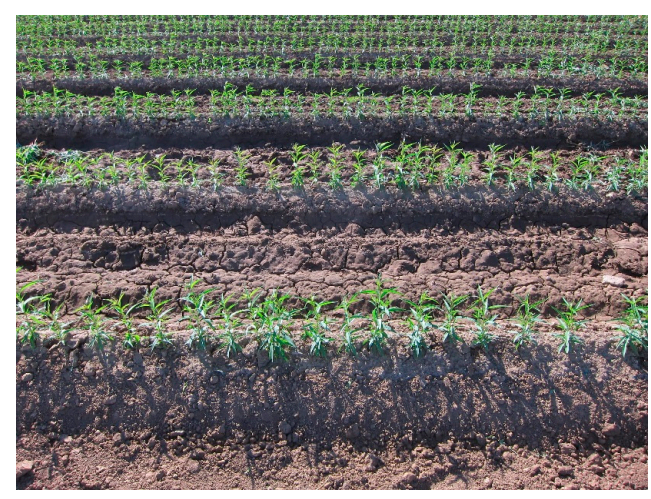

Figure 8. Sample image of rows for the purpose of counting multiple rows simultaneously.

\section{Acknowledgments}

Authors would like to thank Sierra Gold Nurseries, Cliff Beumel the Vice President of Sierra Gold Nurseries, and Rashpal Uppal their Production Manager for their assistance in conducting this research. We would also like to thank Burt Vannucci, Department of Biological and Agricultural Engineering, University of California, Davis, who helped build and test the system. This work was supported in part by the USDA National Institute of Food and Agriculture Hatch/Multistate project CA-D-BAE-7808-RR. Any opinions, findings, conclusions, or recommendations expressed in this publication are those of the author(s) and do not necessarily reflect the view of the National Institute of Food and Agriculture (NIFA) or the United States Department of Agriculture (USDA). 


\section{Author Contributions}

David Slaughter and Bradley Hanson are the project leaders. Hanson was in charge of the field site coordination with Sierra Gold Nurseries and all aspects related to tree crop production and biology. Slaughter was in charge of the overall design of the mobile platform, computer vision hardware and general imaging processing approach and project management. The students, Andrew Barber, Amy Freitas, Daniel Robles, and Erin Whelan, were responsible for the detailed design of the platform. Burt Vannucci was system fabrication team leader and in charge of all equipment logistics, and the students performed the system fabrication. Erin and Daniel were in charge of vehicle operation. Andrew and Amy were in charge of the camera control system and the odometry sensor. Andrew was also the student team design leader. Slaughter, Vannucci, Barber, Freitas, Robles, and Whelan collected all the field data, and the students also made in-field ground truth counts. Thuy Tuong Nguyen was in charge of developing computer vision and image processing algorithms, writing the manuscript, and making image based ground truth counts. Sierra Gold Nurseries produced the crop and allowed researchers access to the tree nursery to test the system.

\section{Conflicts of Interest}

The authors declare no conflict of interest.

\section{References}

1. Pajares, G.; Peruzzi, A.; Gonzalez-de-Soto, P. Sensors in agriculture and forestry. Sensors 2013, 13, 12132-12139.

2. Emmi, L.; Gonzalez-de-Soto, M.; Pajares, G.; Gonzalez-de-Soto, P. Integrating sensory/actuation systems in agricultural vehicles. Sensors 2014, 14, 4014-4049.

3. Fernández, R.; Salinas, C.; Montes, H.; Sarria, J. Multisensory system for fruit harvesting robots. Experimental testing in natural scenarios and with different kinds of crops. Sensors 2014, 14, 23885-23904.

4. Shrestha, D.S.; Steward, B.L. Automatic corn plant population measurement using machine vision. Trans. ASAE 2003, 46, 559-565.

5. Tang, L.; Tian, L.F. Plant identification in mosaicked crop row images for automatic emerged corn plant spacing measurement. Trans. ASABE 2008, 51, 2181-2191.

6. Wang, C..; Guo, X.; Zhao, C. Detection of corn plant population and row spacing using computer vision. In Proceedings of the International Conference on Digital Manufacturing and Automation, Zhangjiajie, China, 5-7 August 2011; pp. 405-408.

7. Garrido, M.; Perez-Ruiz, M.; Valero, C.; Gliever, C.J.; Hanson, B.D.; Slaughter, D.C. Active optical sensors for tree stem detection and classification in nurseries. Sensors 2014, 14, 10783-10803.

8. Bazi, Y.; Al-Sharari, H.; Melgani, F. An automatic method for counting olive trees in very high spatial remote sensing images. In Proceedings of the IEEE International Geoscience and Remote Sensing Symposium, Cape Town, South Africa, 12-17 July 2009; pp. II-125-II-128. 
9. Bazi, Y.; Malek, S.; Alajlan, N.; AlHichri, H. An automatic approach for palm tree counting in UAV images. In Proceedings of the IEEE International Geoscience and Remote Sensing Symposium, Quebec, QC, Canada, 13-18 July 2014; pp. 537-540.

10. Kumar, D.G.; Padmaja, M. A novel image processing technique for counting the number of trees in a satellite image. Eur. J. Appl. Eng. Sci. Res. 2012, 1, 151-159.

11. Bay, H.; Ess, A.; Tuytelaars, T.; van Gool, L. SURF: Speeded up Robust Features. Comput. Vis. Image Underst. 2008, 110, 346-359.

12. Muja, M.; Lowe, D.G. Scalable nearest neighbor algorithms for high dimensional data. IEEE Trans. Pattern Anal. Mach. Intell. 2014, 36, 2227-2240.

13. Sainz-Costa, N.; Ribeiro, A.; Burgos-Artizzu, X.P.; Guijarro, M.; Pajares, G. Mapping wide row crops with video sequences acquired from a tractor moving at treatment speed. Sensors 2011, 11, 7095-7109.

14. Nguyen, T.T.; Pham, X.D.; Song, J.H.; Jin, S.-H.; Kim, D.-K.; Jeon, J.W. Compensating background for noise due to camera vibration in uncalibrated-camera-based vehicle speed measurement systems. IEEE Trans. Veh. Technol. 2011, 60, 30-43.

15. Ribeiro, A.; Ranz, J.; Burgos-Artizzu, X.P.; Pajares, G.; Sanchez del Arco, M.J.; Navarrete, L. An image segmentation based on a genetic algorithm for determining soil coverage by crop residues. Sensors 2011, 11, 6480-6492.

16. Reina, G.; Milella, A. Towards autonomous agriculture: Automatic ground detection using trinocular stereovision. Sensors 2012, 12, 12405-12423.

17. Tian, L.; Slaughter, D.C.; Norris, R.F. Outdoor field machine vision identification of tomato seedlings for automated weed control. Trans. ASABE 1997, 40, 1761-1768.

18. Ta, D.-N.; Chen, W.C.; Gelfand, N.; Pulli, K. SURFTrac: Efficient tracking and continuous object recognition using local feature descriptors. In Proceedings of the IEEE International Conference on Computer Vision Pattern Recognition, Miami, FL, USA, 20-25 June 2009.

19. Juan, L.; Gwun, O. SURF applied in panorama image stitching. In Proceedings of the International Conference on Image Processing Theory Tools and Applications, Paris, France, 7-10 July 2010; pp. $459-499$.

20. Segundo, M.P.; Gomes, L.; Bellon, O.R.P.; Silva, L. Automating 3D reconstruction pipeline by SURF-based alignment. In Proceedings of the IEEE International Conference on Image Processing, Orlando, FL, USA, 30 September-3 October 2012; pp. 1761-1764.

21. Nguyen, T.T.; Pham, X.D.; Jeon, J.W. Scaling-translation parameter estimation using Genetic Hough Transform for background compensation. KSII Trans. Internet Inf. Syst. 2011, 5, 1423-1443.

22. Chen, Y.; Zhang, R.H.; Shang, L. A novel method of object detection from a moving camera based on image matching and frame coupling. PLOS ONE 2014, 9, e109809.

(C) 2015 by the authors; licensee MDPI, Basel, Switzerland. This article is an open access article distributed under the terms and conditions of the Creative Commons Attribution license (http://creativecommons.org/licenses/by/4.0/). 